\title{
Charge Pumping in Mesoscopic Systems coupled to a Superconducting Lead
}

\author{
M. Blaauboer \\ Department of Applied Physics, Delft University of Technology, Lorentzweg 1, 2628 CJ Delft, The Netherlands
}

(Dated: November 1, 2018)

\begin{abstract}
We derive a general scattering-matrix formula for the pumped current through a mesoscopic region attached to a normal and a superconducting lead. As applications of this result we calculate the current pumped through (i) a pump in a wire, (ii) a quantum dot in the Coulomb blockade regime, and (iii) a ballistic double-barrier junction, all coupled to a superconducting lead. Andreev reflection is shown to enhance the pumped current by up to a factor of 4 in case of equal coupling to the leads. We find that this enhancement can still be further increased for slightly asymmetric coupling.

PACS numbers: 73.23.-b, 72.10.Bg, 74.80.Fp
\end{abstract}

\section{INTRODUCTION}

It is well-known that the quantum transport properties of a mesoscopic system are modified in the presence of a superconducting interface, due to interference between normal and Andreev reflections. Andreev reflection $(\mathrm{AR})$ is the electron-to-hole reflection process which occurs when an electron with energy slightly above the Fermi energy is incident on the boundary between a normal metal and a superconductor: the electron enters the superconductor after forming a Cooper pair, and leaves a hole in the normal metal with energy slightly below the Fermi level which travels back along (nearly) the same path where the electron came from. Because of the phasecoherent character of AR, it is interesting to study its effect on transport in mesoscopic systems, where phase coherence plays an important role. In the last decade, this has led to the discovery of a wealth of quantum interference effects in mesoscopic normal-metal-superconductor (NS) structurest, such as the observation of a large narrow peak in the differential conductance of a disordered NS junction ("reflectionless tunneling") 3 , and the discovery of novel Kondo phenomena in quantum dots attached to a normal and a superconducting leadt. In addition, investigations of the conductance in superconductorcarbon-nanotube devices have recently appeared5, which indicate that also in these devices resonant behavior due to AR occurs.

The purpose of this paper is to study the effects of AR on a new type of mesoscopic transport, namely adiabatic quantum pumping. Quantum pumping involves the generation of a d.c. current in the absence of a bias voltage by periodic modulations of two or more system parameters, such as e.g. the shape of the system or a magnetic field. The idea was pioneered by Thouless for electrons moving in an infinite one-dimensional periodic potential la $^{6}$. In recent years, adiabatic quantum pumpine in quantum dots has attracted a lot of attentiond is. Quantum dots are small metallic or semiconducting islands, confined by gates and connected to electron reservoirs (leads) through quantum point contacts (QPCs) 10 . In addition to investigations of pumping in quantum dots, theoretical ideas have been put forward for charge pump- ing ipcarbon nanotubes 11 , and for pumping of Cooper pair12. Here we consider a mesoscopic system consisting of an arbitrary normal-metal region, e.g. a quantum dot or QPC, coupled to a superconductor, as schematically depicted in Fig. 1(a). We start by deriving a general formula for the pumped current through this NS system in terms of its scattering matrix (Sec. II). This is the $N$ mode generalization of the result of Wang et al. for a NS system with single-mode leads 13 . We then use this result to calculate the current pumped through: a simple peristaltic pump (Sec. III A), a quantum dot in the Coulomb blockade regime (Sec. [II B), and a double-barrier junction (Sec. III C), each coupled to a superconducting lead. Comparing these with the pumped current in the corresponding systems attached to normal leads only, shows that AR enhances quantum pumping by up to a factor of $>4$ in systems with (nearly) symmetric coupling to the leads, while it reduces quantum pumping in the opposite situation of strongly asymmetric coupling.

\section{DERIVATION OF NS PUMPING FORMULA}

Consider the system in Fig. 11(a). The normal region (which may e.g. be disordered, or contain a constriction) is coupled to ideal normal leads 1 and 2 containing $N$ modes each. No bias voltage is applied to the system, so all reservoirs are held at the same potential. We assume a constant pair potential $\Delta(\vec{r})=\Delta_{0} e^{i \phi}$ in the superconductor, which is applicable for wide junctions 44 and has previously been used to derive the conductance through a NS junction 15 . We also assume that the NS interface is ideal, i.e. no specular reflection occurs for energies $0<\epsilon<\Delta_{0}$, with $\epsilon$ the energy measured from the Fermi energy $\epsilon_{F}$. The scattering matrix $S_{\mathrm{NS}}$ of the entire system is given by 16

$$
S_{\mathrm{NS}}(\epsilon)=\left(\begin{array}{cc}
S^{e e}(\epsilon) & S^{e h}(\epsilon) \\
S^{h e}(\epsilon) & S^{h h}(\epsilon)
\end{array}\right)
$$


where $S^{e e}-S^{h h}$ are $N \times N$ scattering matrices given by

$$
\begin{aligned}
S^{e e}(\epsilon) & =r_{11}(\epsilon)+\alpha^{2} t_{12}(\epsilon) r_{22}^{*}(-\epsilon) M_{e} t_{21}(\epsilon) \\
S^{e h}(\epsilon) & =\alpha e^{i \phi} t_{12}(\epsilon) M_{h} t_{21}^{*}(-\epsilon) \\
S^{h e}(\epsilon) & =\alpha e^{-i \phi} t_{12}^{*}(-\epsilon) M_{e} t_{21}(\epsilon) \\
S^{h h}(\epsilon) & =r_{11}^{*}(-\epsilon)+\alpha^{2} t_{12}^{*}(-\epsilon) r_{22}(\epsilon) M_{h} t_{21}^{*}(-\epsilon) .
\end{aligned}
$$

Here $r_{i i}(\epsilon)\left[t_{i j}(\epsilon)\right], i, j=1,2$, denotes the reflection [transmission] amplitude for electrons at energy $\epsilon$, with $0<\epsilon<\Delta_{0}$, traveling from lead $i[j]$ to lead $i$, $\alpha \equiv \exp \left[-i \arccos \left(\epsilon / \Delta_{0}\right)\right], M_{e} \equiv\left[1-\alpha^{2} r_{22}(\epsilon) r_{22}^{*}(-\epsilon)\right]^{-1}$, and $M_{h} \equiv\left[1-\alpha^{2} r_{22}^{*}(-\epsilon) r_{22}(\epsilon)\right]^{-1}$. The scattering matrix (11) is unitary and satisfies the symmetry relation $S_{\mathrm{NS}}(\epsilon, B, \phi)_{i j}=S_{\mathrm{NS}}(\epsilon,-B,-\phi)_{j i}$ for time reversal invariance. Adiabatic quantum pumping in this NS junction is obtained by slow and periodic variations of two external parameters $X_{1}$ and $X_{2}$ as $X_{1}(t)=\bar{X}_{1}+\delta X_{1} \sin (\omega t)$ and $X_{2}(t)=\bar{X}_{2}+\delta X_{2} \sin (\omega t+\phi)$. The frequency $\omega$ has to be such that $\omega \ll \tau_{\text {dwell }}^{-1}$, with $\tau_{\text {dwell }}$ the time particles spend in the system, in order for equilibrium to be maintained throughout the entire pumping cycle. The net charge $\delta Q(t)$ emitted into lead 1 due to the modulations $\delta X_{1}$ and $\delta X_{2}$ consists of the amount of negative charge carriers (electrons) minus the amount of positive charge carriers (holes) emitted into lead 1. For infinitesimal variations $\delta X_{i}, i=1,2$, this charge is given by

$$
\begin{aligned}
\delta Q(t)= & \frac{e}{2 \pi} \sum_{\alpha, \beta \in 1}\left[\operatorname{Im}\left(\frac{\partial S_{\alpha \beta}^{e e}}{\partial X_{1}} S_{\alpha \beta}^{e e *}-\frac{\partial S_{\alpha \beta}^{h e}}{\partial X_{1}} S_{\alpha \beta}^{h e *}\right) \delta X_{1}(t)\right. \\
& \left.+\operatorname{Im}\left(\frac{\partial S_{\alpha \beta}^{e e}}{\partial X_{2}} S_{\alpha \beta}^{e e *}-\frac{\partial S_{\alpha \beta}^{h e}}{\partial X_{2}} S_{\alpha \beta}^{h e *}\right) \delta X_{2}(t)\right], \quad(3)
\end{aligned}
$$

where the indices $\alpha$ and $\beta$ are summed over all $N$ modes in lead 1. This expression is obtained along the same lines as the pumped current in a quantum dot coupled to two normal leads and based on a formula derived by Büttiker et al.17, see also Ref.13. The total charge emitted into lead 1 during one period $\tau \equiv 2 \pi / \omega$ is found by integrating Eq. (3) over time,

$$
\begin{aligned}
Q(\tau)= & \frac{e}{2 \pi} \int_{0}^{\tau} d t \sum_{\alpha, \beta \in 1}\left[\operatorname{Im}\left(\frac{\partial S_{\alpha \beta}^{e e}}{\partial X_{1}} S_{\alpha \beta}^{e e *}-\frac{\partial S_{\alpha \beta}^{h e}}{\partial X_{1}} S_{\alpha \beta}^{h e *}\right)\right. \\
& \left.\frac{d X_{1}}{d t}+\operatorname{Im}\left(\frac{\partial S_{\alpha \beta}^{e e}}{\partial X_{2}} S_{\alpha \beta}^{e e *}-\frac{\partial S_{\alpha \beta}^{h e}}{\partial X_{2}} S_{\alpha \beta}^{h e *}\right) \frac{d X_{2}}{d t}\right]
\end{aligned}
$$

and rewriting this as an integral over the area $A$ that is enclosed in parameter space $\left(X_{1}, X_{2}\right)$ during one period. We then find that the total current $I_{\mathrm{NS}} \equiv \frac{\omega}{2 \pi} Q(\tau)$ pumped into lead 1 is given by

$$
\begin{aligned}
I_{\mathrm{NS}} & =\frac{\omega e}{2 \pi^{2}} \int_{A} d X_{1} d X_{2} \sum_{\alpha, \beta \in 1} \Pi_{\alpha \beta}\left(X_{1}, X_{2}\right) \\
& \approx \frac{\omega e}{2 \pi} \delta X_{1} \delta X_{2} \sin \phi \sum_{\alpha, \beta \in 1} \Pi_{\alpha \beta}\left(X_{1}, X_{2}\right),
\end{aligned}
$$

(a)

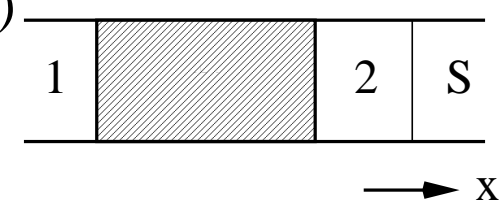

(b)

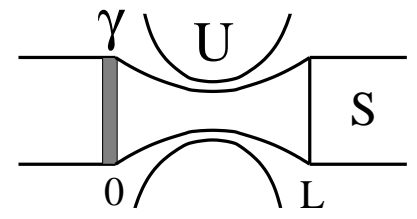

(c)

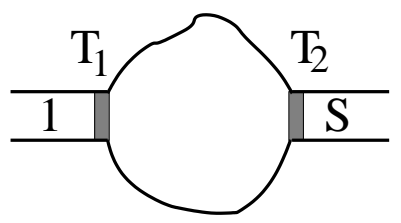

FIG. 1: (a) Normal-metal region (hatched) adjacent to a superconducting lead (S). The normal leads 1 and 2 contain $\mathrm{N}$ modes each. (b) NS pump in a $1 \mathrm{D}$ wire containing a tunnel barrier $\gamma$ and an external potential $U$. (c) Quantum dot coupled via tunneling barriers $T_{1}$ and $T_{2}$ to a normal (1) and a superconducting (S) lead.

with

$$
\Pi_{\alpha \beta}\left(X_{1}, X_{2}\right) \equiv \operatorname{Im}\left[\frac{\partial S_{\alpha \beta}^{e e *}}{\partial X_{1}} \frac{\partial S_{\alpha \beta}^{e e}}{\partial X_{2}}-\frac{\partial S_{\alpha \beta}^{h e *}}{\partial X_{1}} \frac{\partial S_{\alpha \beta}^{h e}}{\partial X_{2}}\right]
$$

Eq. (4) is valid at zero temperature and to first order in the frequency $\omega_{\text {- For }} N=1$ it reduces to the singlemode result of Ref 13 (apart from a factor of 2 for spin degeneracy in the latter). Eq. (4b) applies for bilinear response in the parameters $X_{1}$ and $X_{2}$, in which case the integral in (4a) becomes independent of the pumping contour. $I_{\mathrm{NS}}$ is of similar generality as the expression for the pumped current in the presence of two normal-metal leadst

$$
I_{\mathrm{N}}=\frac{\omega e}{2 \pi^{2}} \int_{A} d X_{1} d X_{2} \sum_{\alpha \in 1} \sum_{\beta \in\{1,2\}} \operatorname{Im}\left(\frac{\partial S_{\alpha \beta}^{*}}{\partial X_{1}} \frac{\partial S_{\alpha \beta}}{\partial X_{2}}\right) .
$$

Here $S_{\alpha \beta}$ denotes the $2 N \times 2 N$ scattering matrix of the system. Note that the index $\beta$ in this case is summed over the modes in both lead 1 and lead 2 , since electrons can be incident from either lead. In our NS junction, the charge pumped into the right lead is converted into a supercurrent.

In order to illustrate the result (4), we now proceed to apply it to several NS configurations. Unless otherwise noted, we restrict ourselves to the linear response regime corresponding to weak pumping. In that regime, only the scattering matrix at the Fermi level $\epsilon=0$ is needed. 


\section{APPLICATIONS}

\section{A. Peristaltic pump in a one-dimensional (1D) wire}

As a first example, consider a "peristaltic" pump formed by a $1 \mathrm{D}$ wire in contact with a superconducting lead, see Fig. 1 (b). The pump is operated by periodically opening and closing a tunnel barrier of height $\gamma$ and varying an external potential $U$. Calculating the scattering matrix (11) from the Schrödinger equation with potential $V(x)=\gamma \delta(x)+U \Theta[x(L-x)]$ and substituting into Eq. (4b) yields the current

$$
I_{\mathrm{NS}}=\frac{3 \sqrt{2}}{32} \frac{\omega e}{\pi k^{2}} \delta U \sin ^{2}(k L) .
$$

Comparing this with the analogous expression in the case of two normal leadst,

$$
I_{\mathrm{N}}=\frac{\omega e L}{8 \pi^{2} k} \delta U+\frac{\omega e}{16 \pi^{2} k^{2}} \delta U\left(\pi \sin ^{2}(k L)-\sin (2 k L)\right),
$$

we see that the presence of AR can both enhance and reduce the pumped current. Maximum enhancement of $I_{\mathrm{NS}} / I_{\mathrm{N}}=3 \sqrt{2} / 2 \approx 2.1$ occurs for short wires such that $2 k L \ll 1$. Note that $I_{\mathrm{NS}}$, unlike $I_{\mathrm{N}}$, has no classical contribution and is entirely due to quantum interference in the wire.

\section{B. Pumped current peak heights in a nearly-isolated quantum dot}

As a second example, we study quantum pumping in a quantum dot which is coupled via two tunneling barriers with transmission probabilities $T_{1}$ and $T_{2}$ to a normal and a superconducting single-mode lead, see Fig. 11(c). Pumping is achieved by periodic variations of the strength of the two tunneling barriers $V_{1}$ and $V_{2}$ (with $V_{1,2} \equiv \sqrt{\left(1-T_{1,2}\right) / T_{1,2}}$ for delta-function barriers) as $V_{1}(t)=V_{1}+\delta V_{1} \sin (\omega t)$ and $V_{2}(t)=\bar{V}_{2}+\delta V_{2} \sin (\omega t+\phi)$. We are interested in the regime of high barriers (where transmission is low, $T_{1}, T_{2} \ll 1$ ), and weak pumping $\left(\delta V_{m} \ll \bar{V}_{m}, m=1,2\right)$. At low temperatures such that $k_{B} T \ll \Delta<E_{C}$ [with $\Delta$ the single-particle level spacing and $E_{C}=e^{2} / C$ the charging energy of the dot, $C$ being the total capacitance] the quantum dot then remains in the Coulomb blockade regime during the whole pumping cycle and transport through the dot is mediated by resonant transmission through a single 18 . Substituting the appropriate scattering matrix 190 into Eq. (4b) yields, up to lowest order in $T_{1}$ and $T_{2}$ and for thermal energies less than the total decay width $\Gamma$ into the leads $k_{B} T<\Gamma \ll \Delta$ [with $\Gamma=\Gamma_{1}+\Gamma_{2} \equiv \hbar \nu\left(T_{1}+T_{2}\right)$ and $\nu$ the attempt frequency, the inverse of the round-trip travel time between the two barriers],

$$
I_{\mathrm{NS}}=\frac{\omega e}{4 \pi} \delta V_{1} \delta V_{2} \sin \phi \frac{T_{1}^{7 / 2} T_{2}^{5 / 2}\left(\sqrt{T_{1}}+\sqrt{T_{2}}\right)}{\left[\frac{1}{4}\left(T_{1}^{2}+T_{2}^{2}\right)+\left(\frac{\epsilon-\epsilon_{\mathrm{res}}}{\hbar \nu}\right)^{2}\right]^{3}} .
$$

Here $\epsilon_{\text {res }}$ denotes the resonance energy for a completely isolated $\operatorname{dot}\left(T_{1}=T_{2}=0\right)$. Note that Eq. (9) is not symmetric with respect to $T_{1}$ and $T_{2}$, in contrast with the conductance $15 G_{\mathrm{NS}}=\frac{e^{2}}{h} \frac{T_{1}^{2} T_{2}^{2}}{\left[\frac{1}{4}\left(T_{1}^{2}+T_{2}^{2}\right)+\left(\frac{\epsilon-\epsilon_{\mathrm{res}}}{\hbar \nu}\right)^{2}\right]^{2}}$ through this system. This is due to the fact that $I_{\mathrm{NS}}$ depends on $\partial S / \partial X$, whereas $G_{\mathrm{NS}}$ depends on the transmission eigenvalues, the eigenvalues of the matrix $t_{12}^{\dagger} t_{12}$. Compared to the pumped current in a dot coupled to two normal leadst 19

$$
I_{\mathrm{N}}=\frac{\omega e}{4 \pi} \delta V_{1} \delta V_{2} \sin \phi \frac{\left(T_{1} T_{2}\right)^{3 / 2}\left(\sqrt{T_{1}}+\sqrt{T_{2}}\right)\left(T_{1}+T_{2}\right)}{\left[\frac{1}{4}\left(T_{1}+T_{2}\right)^{2}+\left(\frac{\epsilon-\epsilon_{\text {res }}}{\hbar \nu}\right)^{2}\right]^{2}}(10)
$$

we find that

$$
\frac{I_{\mathrm{NS}}}{I_{\mathrm{N}}}=\frac{T_{1}^{2} T_{2}\left[\frac{1}{4}\left(T_{1}+T_{2}\right)^{2}+\left(\frac{\epsilon-\epsilon_{\mathrm{res}}}{\hbar \nu}\right)^{2}\right]^{2}}{\left(T_{1}+T_{2}\right)\left[\frac{1}{4}\left(T_{1}^{2}+T_{2}^{2}\right)+\left(\frac{\epsilon-\epsilon_{\mathrm{res}}}{\hbar \nu}\right)^{2}\right]^{3}}
$$

For symmetric coupling $\left(T_{1}=T_{2}\right)$ close to resonance, $\left(\epsilon-\epsilon_{\text {res }}\right) \ll \hbar \nu T_{1,2} \equiv \Gamma_{1,2}$, AR enhances the pumped current by a factor of 421 . In case of strongly asymmetric coupling close to resonance, on the other hand, AR reduces the pumped amplitude: $I_{\mathrm{NS}} / I_{\mathrm{N}} \sim\left(T_{1} / T_{2}\right)^{2} \ll 1$ for $T_{1} \ll T_{2}$, and $I_{\mathrm{NS}} / I_{\mathrm{N}} \sim T_{2} / T_{1} \ll 1$ for $T_{2} \ll T_{1}$. The enhancement by a factor of 4 for symmetric barriers consists of 2 contributions of a factor of 2: one factor of 2 is due to the contribution of both electrons and holes to the current, which is also responsible for the dopbling of conductance $G_{\mathrm{NS}} / G_{\mathrm{N}}=2$ in this NS structure15. A second factor of 2 comes from the asymmetry of the NS dot with respect to injection of charge carriers into the leads, since electrons can only leave the system through the left, normal lead. This leads to an extra doubling of the pumped current compared to the normal case where electrons are injected into both the left and the right leads. This extra factor of 2 does not occur in the presence of an applied bias, as for conductance, since the bias causes charge carriers to flow from one side to the other in both the normal and the NS system. Note that due to the asymmetry of Eq. (11) with respect to $T_{1}$ and $T_{2}$ the maximum attainable enhancement is even larger than 4: for a slightly asymmetric junction (with $T_{1} / T_{2} \sim 1.26$ ) one obtains $I_{\mathrm{NS}} / I_{\mathrm{N}} \sim 4.23$. In this case quantum interference between electrons and holes in the NS system is maximal. If the barrier asymmetry is further increased, $I_{\mathrm{NS}} / I_{\mathrm{N}}$ decreases and eventually becomes less than 1 for strongly asymmetric coupling, when pumping is dominated by one barrier only.

At temperatures higher than the decay width, $\Gamma \ll k_{B} T \ll \Delta$, the pumped current exhibits Coulomb oscillations as a function of an applied gate voltage 19. The peak heights of these oscillations can be obtained by thermally averaging Eq. (9) as $I_{\mathrm{NS}, \text { peak }} \equiv-\int d \epsilon I_{\mathrm{NS}} f^{\prime}(\epsilon, T) \approx \frac{1}{4 k_{B} T} \int_{\epsilon_{\mathrm{res}}-\frac{1}{2} \hbar \nu}^{\epsilon_{\mathrm{res}}} \sqrt{T_{1}^{2}+T_{2}^{2}} d \epsilon I_{\mathrm{NS}}$, where $f(\epsilon, T) \equiv\left[1+\exp \left(\epsilon / k_{B} T\right)\right]^{-1}$ denotes the Fermi 
function. We obtain

$$
I_{\text {NS,peak }}=\frac{\omega e(8+3 \pi) \hbar \nu}{16 \pi k_{B} T} \delta V_{1} \delta V_{2} \sin \phi \frac{T_{1}^{\frac{7}{2}} T_{2}^{\frac{5}{2}}\left(T_{1}^{\frac{1}{2}}+T_{2}^{\frac{1}{2}}\right)}{\left(T_{1}^{2}+T_{2}^{2}\right)^{\frac{5}{2}}}(12)
$$

This thermal average does not explicitly include the effect of the charging energy $E_{C}$ on the pumped current. A full linear response theory for Coulomb blockade conductance oscillations including charging energy was developed in Ref.18. There it was shown that for temperatures $k_{B} T \ll \Delta$ only one level $N_{\text {min }}$ participates in the transport $\left[N_{\min }\right.$ is defined as the level which minimizes the energy $E_{\mathrm{N}}+\mathrm{U}(\mathrm{N})-\mathrm{U}(\mathrm{N}-1)-\epsilon_{\mathrm{F}}$, with $E_{\mathrm{N}}$ the energy of the Nth level of the dot, and $\mathrm{U}(\mathrm{N})$ the electrostatic energy of a dot containing $\mathrm{N}$ electrons], and the oscillation peaks are well described by the thermal average. One can show that for the same reason the pumped current peaks in this temperature range are well described by the thermal average (12), with the understanding that $T_{1,2}$ in Eq. (12) refer to the level $A$ min. From (12) and the analogous normal-state result 19

$I_{\mathrm{N}, \text { peak }}=\frac{\omega e(2+\pi) \hbar \nu}{16 \pi k_{B} T} \delta V_{1} \delta V_{2} \sin \phi \frac{\left(T_{1} T_{2}\right)^{\frac{3}{2}}\left(T_{1}^{\frac{1}{2}}+T_{2}^{\frac{1}{2}}\right)}{\left(T_{1}+T_{2}\right)^{2}}$

we obtain

$$
\frac{I_{\mathrm{NS}, \text { peak }}}{I_{\mathrm{N}, \text { peak }}}=\left(\frac{8+3 \pi}{2+\pi}\right) \frac{T_{1}^{2} T_{2}\left(T_{1}+T_{2}\right)^{2}}{\left(T_{1}^{2}+T_{2}^{2}\right)^{5 / 2}} .
$$

Also here, AR enhances the pumped current in case of symmetric tunnel barriers, while a reduction occurs for asymmetric barriers. Maximum enhancement of $I_{\mathrm{NS}, \text { peak }} / I_{\mathrm{N} \text {,peak }} \sim 2.55$ is reached for $T_{1} / T_{2} \sim 1.292$. This factor is less than 4 , because the average over energy $\epsilon$ from which the peak heights (12) are obtained also involves contributions of $I_{\mathrm{NS}}$ [Eq. (9)] further away from resonance, for which $I_{\mathrm{NS}} / I_{\mathrm{N}}$ is much less than 4 [consider e.g. Eq. (11) for $\left.\epsilon-\epsilon_{\mathrm{res}}=\frac{1}{2} \hbar \nu \sqrt{T_{1}^{2}+T_{2}^{2}}\right]$. This results in lower maximal enhancement of the pumped current peaks (14) at higher temperatures $k_{B} T \gg \Gamma$.

Another interesting result is obtained in this system by relaxing the assumption of weak pumping and considering quantum pumping by varying the two tunneling barriers in such a way that the loop which describes the pumping cycle in parameter space encircles the entire resonance line. For normal-metal contacts this problem has recently been studied 20 and led to the prediction that at zero temperature the charge transferred during one pumping cycle is quantized, $Q=e$ (for spinless electrons). The transferred charge in our NS system is obtained by substituting the scattering matrix of a 1D double-barrier junction given in Ref 19 into Eq. (4a) and integrating over the resonance line $V_{1}^{-1}+V_{2}^{-1}=$ $\left|\left(\epsilon-\epsilon_{\mathrm{res}}\right) / \hbar \nu\right| \ll 1$, with $V_{i}^{-1} \equiv \sqrt{T_{i} /\left(1-T_{i}\right)}$ for $i=1,2$. We then obtain

$$
Q=\frac{3 \sqrt{2} e}{2} \int_{-1}^{1} d z \frac{(1+z)^{2}\left(1-z^{2}\right)^{3}}{\left[1+6 z^{2}+z^{4}\right]^{5 / 2}}=e,
$$

so AR neither enhances nor reduces quantum pumping. This occurs because charge is effectively transferred by a shuttle mechanism (first through one barrier and then through the next), which is unaffected by Andreev interference effects and fixed by the pumping loop. Since only pairs of electrons can enter the superconductor, but the strong Coulomb interaction (charging energy) forbids simultaneous pumping of 2 electrons with opposite spin, this pumping process is not allowed in a nearly-closed NS quantum dot. As pointed out in Ref.22 it can, however, occur in a double-barrier junction in which electronelectron interactions may be neglected. Both in case of two normal and in case of one normal and one superconducting single-mode lead a quantized amount of charge of $2 \mathrm{e}$ is then transferred during each pumping cycle 22 .

\section{Ballistic double-barrier junction}

Finally, we compare the pumped current $I_{\mathrm{N}}$ in a $N I_{1} N I_{2} N$ junction vs. $I_{\mathrm{NS}}$ in a $N I_{1} N I_{2} S$ junction, where $I_{1,2}$ denote tunnel barriers with transmission probability per mode $T_{1,2}$, see inset of Fig. 2. In linear response, assuming $N$-mode leads and ballistic transport between the barriers, the pumped currents are given by

$$
I_{\mathrm{N}}=I_{\mathrm{C}} T_{1}^{\frac{3}{2}} T_{2}^{\frac{3}{2}} \sum_{n=1}^{N} \frac{A_{\mathrm{N}}+B_{\mathrm{N}} \cos \phi_{n}+C_{\mathrm{N}} \sin \phi_{n}}{\left(D_{\mathrm{N}}+E_{\mathrm{N}} \cos \phi_{n}\right)^{2}},
$$

and

$$
I_{\mathrm{NS}}=4 I_{\mathrm{C}} T_{1}^{3} T_{2}^{\frac{5}{2}} \sum_{n=1}^{N} \frac{A_{\mathrm{NS}}+B_{\mathrm{NS}} \cos \phi_{n}+C_{\mathrm{NS}} \sin \phi_{n}}{\left(D_{\mathrm{NS}}+E_{\mathrm{NS}} \cos \phi_{n}\right)^{3}}
$$

Here $I_{\mathrm{C}} \equiv \frac{\omega e}{2 \pi} \delta V_{1} \delta V_{2} \sin \phi 23$, and $A_{\mathrm{N}}, \ldots, E_{\mathrm{NS}}$ are given by $A_{\mathrm{N}} \equiv \sqrt{R_{1} T_{2}}+\sqrt{R_{2} T_{1}}, B_{\mathrm{N}} \equiv-\sqrt{R_{1} R_{2}}\left(\sqrt{R_{1} T_{2}}+\right.$ $\left.\sqrt{R_{2} T_{1}}\right), C_{\mathrm{N}} \equiv-1+R_{1} R_{2}-\sqrt{R_{1} R_{2} T_{1} T_{2}}, D_{\mathrm{N}} \equiv 1+R_{1} R_{2}$, $E_{\mathrm{N}} \equiv-2 \sqrt{R_{1} R_{2}}, A_{\mathrm{NS}} \equiv 2 \sqrt{R_{2}}, B_{\mathrm{NS}} \equiv \sqrt{R_{2} T_{1} T_{2}}-$ $\sqrt{R_{1}}\left(2-T_{2}\right), C_{\mathrm{NS}} \equiv-\left(\sqrt{T_{1}}\left(2-T_{2}\right)+\sqrt{R_{1} R_{2} T_{2}}\right), D_{\mathrm{NS}} \equiv$ $\left(1+R_{1}\right)\left(1+R_{2}\right)$, and $E_{\mathrm{NS}} \equiv-4 \sqrt{R_{1} R_{2}}$. For $L \gg \lambda_{F}$, with $\lambda_{F}$ the Fermi wavelength, and $N T_{i} \gg 1$ the current is not dominated by a single resonance and the phases $\phi_{n}$ are uniformly distributed from 0 to $2 \pi$. Replacing the sums in Eqs. (16) and (17) by integrals over $\phi_{n}$ then yields the average pumped currents

$$
\begin{aligned}
\left\langle I_{\mathrm{N}}\right\rangle= & I_{\mathrm{C}} \frac{T_{1}^{\frac{3}{2}} T_{2}^{\frac{3}{2}}}{\pi\left(1-R_{1} R_{2}\right)^{2}}\left[2 \left(-1+R_{1} R_{2}+\right.\right. \\
& \left.\left.\sqrt{R_{1} R_{2} T_{1} T_{2}}\right)-\pi\left(\sqrt{R_{1} T_{2}}+\sqrt{R_{2} T_{1}}\right)\right],
\end{aligned}
$$

and

$$
\begin{aligned}
\left\langle I_{\mathrm{NS}}\right\rangle & =4 I_{\mathrm{C}} \frac{T_{1}^{3} T_{2}^{\frac{5}{2}}}{\pi\left(D_{\mathrm{NS}}^{2}-E_{\mathrm{NS}}^{2}\right)^{\frac{5}{2}}}\left[2 C_{\mathrm{NS}} D_{\mathrm{NS}}\left(D_{\mathrm{NS}}^{2}-E_{\mathrm{NS}}^{2}\right)^{\frac{1}{2}}\right. \\
& \left.-A_{\mathrm{NS}}\left(E_{\mathrm{NS}}^{2}+2 D_{\mathrm{NS}}^{2}\right) / 2+3 B_{\mathrm{NS}} D_{\mathrm{NS}} E_{\mathrm{NS}} / 2\right]
\end{aligned}
$$




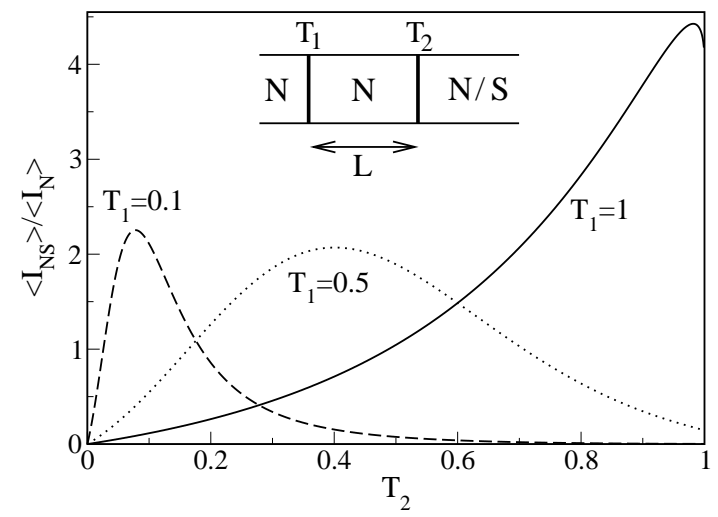

FIG. 2: Ratio of the average pumped currents $\left\langle I_{N S}\right\rangle$ and $\left\langle I_{N}\right\rangle$ in ballistic NININ and NINIS junctions as a function of $T_{2}$ for $T_{1}=1,0.5$, and 0.1 . The inset shows the double-barrier junction considered.

whose ratio is plotted in Fig. 2. As for a nearly-isolated quantum dot, $\left\langle I_{N S}\right\rangle /\left\langle I_{N}\right\rangle$ is largest in case of nearlysymmetric coupling. Only for $T_{1}=1$ the maximum enhancement by a factor of $>4$ is obtained, since for $T_{1}<1$ less electrons reach the NS interface due to normal reflections at the barriers, which in the absence of resonances reduces the effect of Andreev reflection.

\section{CONCLUSION}

In conclusion, we have studied adiabatic quantum pumping in mesoscopic NS systems. Compared to the conductance in these systems we predict two striking differences: (1) For a nearly-isolated quantum dot with symmetric $\left(T_{1}=T_{2}\right)$ tunneling barriers, and a transparent $\left(T_{1}=T_{2}=1\right)$ double-barrier NS junction, AR enhances the pumped current by a factor of 4 , which is twice the maximum enhancement of the conductance in these systems. (2) In case of quantum pumping this enhancement is not an absolute maximum, whereas in case of conductance it is. These differences are due to, resp., the absence of an external bias and the asymmetric dependence on the tunnel barriers in case of quantum pumping. We hope that these fascinating effects of Andreev reflection on quantum pumping will find experimental confirmation, f.g. in present-day available nearly-closed quantum dots24. Stimulating discussions with C.M. Marcus, N.W.A. Tollenaar and Yu. V. Nazarov are gratefully acknowledged. This work was supported by the Stichting voor Fundamenteel Onderzoek der Materie (FOM), by NFS grant CHE-0073544 and by an NSF MRSEC grant.
1 A.F. Andreev, Zh. Eksp. Teor. Fiz. 46, 1823 (1964) [Sov. Phys. JETP 19, 1228 (1964)]; ibid. 51, 1510 (1966) [24, 1019 (1967)].

${ }^{2}$ C.W.J. Beenakker, Rev. Mod. Phys. 69, 731 (1997).

3 A. Kastalsky et al., Phys. Rev. Lett. 67, 3026 (1991); A.F. Volkov et al., Physica C 210, 21 (1993); Yu. V. Nazarov, Phys. Rev. Lett. 73, 134 (1994); ibid., 1420 (1994); C.W.J. Beenakker et al., Phys. Rev. Lett. 72, 2470 (1994).

${ }^{4}$ K. Kang, Phys. Rev. B 58, 9641 (1998); A.A. Clerk et al., ibid. 61, 3555 (2000); J.C. Cuevas et al.,ibid. 63, 094515 (2001); Q. Sun et al., Phys. Rev. Lett. 87, 176601 (2001).

5 A.F. Morpurgo et al., Science 286, 263 (1999); Y. Wei et al., Phys. Rev B 63, 195412 (2001).

6 D.J. Thouless, Phys. Rev. B 27, 6083 (1983).

7 P.W. Brouwer, Phys. Rev. B 58, R10135 (1998).

8 See e.g. I.L. Aleiner and A.V. Andreev, Phys. Rev. Lett. 81, 1286 (1998); F. Zhou et al., Phys. Rev. Lett. 82, 608 (1999); J.E. Avron et al., Phys. Rev. B 62, 10618 (2000); M.G. Vavilov et al., Phys. Rev. B 63, 195313 (2001); J.N.H.J. Cremers and P.W. Brouwer, Phys. Rev. B 65, 115333 (2002).

9 M. Switkes, C.M. Marcus, K. Campman, and A.D. Gossard, Science 283, 1905 (1999).

10 For a review on electron transport through quantum dots see L.P. Kouwenhoven et al., in Proceedings of the Advanced Study Institute on Mesoscopic Electron Transport, L.P. Kouwenhoven, G. Schön, L.L. Sohn, Eds. (Kluwer, Dordrecht, 1997).

11 Y. Wei, J. Wang, H. Guo, and C. Roland, Phys. Rev. B 64, 115321 (2001).

12 F. Zhou, cond-mat/9905190; M. Aunola, Phys. Rev B 63,
132508 (2001).

13 J. Wang, Y. Wei, B. Wang, and H. Guo, Appl. Phys. Lett. 79, 3977 (2001).

14 K.K. Likharev, Rev. Mod. Phys. 51, 101 (1979).

15 C.W.J. Beenakker, Phys. Rev. B 46, 12841 (1992).

16 C.W.J. Beenakker, in Mesoscopic Quantum Physics, Les Houches, Session LXI, edited by E. Akkermans, G. Montambaux, and J.-L. Pichard (North-Holland, Amsterdam, 1994).

17 M. Büttiker, H. Thomas, and A. Prêtre, Z. Phys. B 94, 133 (1994); T. Gramespacher and M. Büttiker, Phys. Rev. B 61, 8125 (2000).

18 C.W.J. Beenakker, Phys. Rev. B 44, 1646 (1991).

19 M. Blaauboer and E.J. Heller, Phys. Rev. B 64, 241301 (2001).

20 Y. Levinson, O. Entin-Wohlman, and P. Wölfle, Physica A 302, 335 (2001).

21 Enhancement by a factor of 4 has recently also been predicted for the ac-conductance in the presence of interactions in a NS wire, see S. Pilgram, H. Schomerus, A.M. Martin, and M. Büttiker, Phys. Rev. B 65, 045321 (2002).

22 Just before submission we became aware of a manuscript by J. Wang et al., condmat/0202333, in which independently a quantized charge of $2 \mathrm{e}$ in a single-mode doublebarrier NS junction is obtained.

23 for spinless electrons, or $I_{\mathrm{C}} \equiv \frac{\omega e}{\pi} \delta V_{1} \delta V_{2} \sin \phi$, taking into account spin degeneracy.

24 J.A. Folk, C.M. Marcus, and J.S. Harris Jr., Phys. Rev. Lett. 87, 206802 (2001). 